\title{
Le curriculum en Finlande : un outil puissant au service de l'éducation
}

Curriculum as a powerful tool of educational development in Finland

El currículum en Finlandia : una poderosa herramienta al servicio de la educación

Irmeli Halinen

\section{OpenEdition}

\section{Journals}

Édition électronique

URL : https://journals.openedition.org/ries/1055

DOI : 10.4000/ries. 1055

ISSN : 2261-4265

Éditeur

France Education international

Édition imprimée

Date de publication : 1 avril 2011

Pagination : 77-88

ISBN : $978-2854205916$

ISSN : 1254-4590

\section{Référence électronique}

Irmeli Halinen, "Le curriculum en Finlande : un outil puissant au service de l'éducation », Revue internationale d'éducation de Sèvres [En ligne], 56 | avril 2011, mis en ligne le 01 avril 2014, consulté le 04 mai 2021. URL : http://journals.openedition.org/ries/1055; DOI : https://doi.org/10.4000/ries.1055

Ce document a été généré automatiquement le 4 mai 2021.

(C) Tous droits réservés 


\title{
Le curriculum en Finlande : un outil puissant au service de l'éducation
}

\author{
Curriculum as a powerful tool of educational development in Finland \\ El currículum en Finlandia : una poderosa herramienta al servicio de la \\ educación
}

Irmeli Halinen

\section{NOTE DE L'ÉDITEUR}

Article traduit de l'anglais par Robert Elbaz et Roger-François Gauthier.

1 Le mot "curriculum» est un mot à signification forte dans le système éducatif finlandais. Son impact s'est clairement renforcé durant les années soixante-dix, lorsque la Finlande a totalement réformé son système d'enseignement général. Cette tendance a également été centrale en matière d'enseignement secondaire supérieur professionnel, enseignement réformé au cours des années quatre-vingt. On peut constater le même processus à l'œuvre durant le début des années 2000, lorsque l'enseignement préscolaire et les activités pré- et post-scolaires pour les élèves relevant de l'enseignement fondamental ont reçu un statut officiel en étant intégrées au système éducatif, obtenant ainsi leur propre curriculum. Ces décennies ont changé le paradigme éducatif en Finlande. Ce développement s'est fondé sur une philosophie bien précise : égalité des chances pour tous en matière d'éducation, haute qualité éducative dans tous les établissements et à tous les niveaux, prise en compte du bien-être de tous les élèves, et enfin confiance accordée aux établissements et aux enseignants. Or on retrouve ces caractéristiques fondatrices - équité et égalité, haute qualité, bien-être et confiance - dans le système curriculaire finlandais lui-même.

Durant la décennie soixante-dix, la Finlande est passée d'un système éducatif, structuré en filières parallèles, fondé sur une sélection précoce des élèves, à un système unifié et ouvert à tous dans lequel l'enseignement de base concerne tous les élèves. Cet 
enseignement s'étale sur neuf ans, et est constitué des précédents niveaux du primaire et du secondaire inférieur. Les municipalités sont pratiquement en charge de la totalité de cet enseignement de base. Il existe en effet fort peu d'écoles d'État et d'écoles privées en Finlande. Après ces neuf années, les élèves peuvent choisir de suivre de façon volontaire une dixième année dans le même type de structure ${ }^{1}$ afin de renforcer leur capacité à suivre des études ultérieures avec succès, ou ils peuvent se diriger directement vers l'enseignement secondaire supérieur, qu'il soit général ou professionnel. Cet enseignement secondaire supérieur dure de trois à quatre ans. Les élèves peuvent ensuite accéder à l'enseignement supérieur, c'est-à-dire aux universités ou aux instituts polytechniques. L'éducation préscolaire volontaire s'adresse aux élèves âgés de 6 ans et dure un an; l'enseignement de base obligatoire commence à l'âge de 7 ans. Les activités volontaires pré- et postscolaires sont spécialement conçues pour les élèves des deux premières années de l'enseignement de base ( 7 et 8 ans) et pour les élèves relevant d'une éducation spéciale dans le cadre de l'enseignement de base. Ces activités visent à donner aux élèves un cadre familier et sûr, dans lequel ils peuvent participer à toute une gamme d'activités stimulantes en dehors des cours. Les soins et l'éducation dont les très jeunes enfants bénéficient au cours des toutes premières années, qui précèdent l'année préscolaire, relèvent, eux, du ministère des Affaires sociales et de la Santé et ne sont pas encore considérés comme faisant officiellement partie du système éducatif. Ce point est susceptible de changer au cours des prochaines années.

3 Aujourd'hui, le système éducatif finlandais est reconnu de façon internationale pour sa très haute qualité. Au cours de quatre évaluations PISA successives comprises entre 2000 et 2009, les élèves de l'école de base ${ }^{2}$ finlandaise ont obtenu d'excellents résultats. Ces résultats indiquent que l'influence du contexte familial est moins marquée en Finlande que dans la moyenne des pays de l'OCDE. Il est tout spécialement remarquable de constater que les écoles finlandaises obtiennent des résultats équitables et que le travail qu'elles fournissent est efficace, alors qu'est consacré un temps modéré à l'apprentissage et avec un budget tout à fait raisonnable (Halinen et Järvinen 2008; Hautamäki et al. 2008). Les enseignants sont hautement qualifiés - le master est obligatoire pour tous les enseignants exerçant dans le cadre de l'école de base et du lycée - et la profession est à la fois valorisée et populaire dans la société finlandaise. Environ $97 \%$ des enfants participent à l'enseignement préscolaire volontaire. Pratiquement tous les élèves obtiennent le certificat de fin d'études fondamentales obligatoires: le pourcentage d'abandon n'est que de $0,3 \%$. D'autre part, lorsqu'ils parviennent au terme de l'enseignement fondamental, $96 \%$ des élèves continuent vers l'éducation secondaire supérieure. En Finlande, une éducation de haute qualité est fortement valorisée. Elle est considérée comme l'un des facteurs les plus importants conditionnant le développement et la réussite de notre avenir (Halinen 2006b).

\section{Le rôle du curriculum et ses évolutions}

4 Aujourd'hui, le curriculum joue un rôle central dans l'organisation et le développement du système éducatif finlandais. Son rôle a changé au cours des années et ces changements sont le reflet des changements de la société. Les trois strates du curriculum - national, municipal et d'établissement - se sont développées en même temps et ont acquis au fil des années des statuts différents. L'interaction entre les 
autorités nationales et municipales d'une part et les personnels scolaires d'autre part a systématiquement augmenté, et une culture de la confiance s'est progressivement construite.

En 1970 a été créé le premier curriculum national pour l'enseignement de base pour tous. Ce curriculum entrait dans les détails alors que le système éducatif était fortement centralisé. Toutes les municipalités et tous les établissements devaient suivre de façon stricte la prescription établie par ce curriculum national. Cette uniformité et cette cohérence dans l'action étaient absolument nécessaires afin de mener à bien cette réforme ambitieuse et de renforcer l'équité et l'égalité du système. Un comité en charge du curriculum de l'école de base (1970) avait été constitué afin concevoir la première partie du curriculum national. Celle-ci incluait les objectifs généraux en matière d'éducation de base, ainsi que les principes pédagogiques et les consignes pratiques destinés à l'enseignement et à l'apprentissage. Il s'agissait là d'une véritable vision à long terme, qui mettait l'accent sur l'égalité des chances de tous en matière d'éducation et qui faisait preuve d'une connaissance approfondie de la pédagogie mais qui, malheureusement, n'a pas fait son chemin dans l'esprit des enseignants et des parents.

6 L'autre partie du curriculum national de 1970, plus connue des enseignants, comprenait l'allocation des heures d'enseignement et le syllabus ${ }^{3}$ détaillé des différentes matières scolaires. La conception de cette partie a été confiée par la loi à un organisme public dédié, central, le Conseil national de l'éducation finlandaise (Finnish national Board of Education: FNBE). Les municipalités devaient élaborer un curriculum "municipal » et chaque établissement devait établir un plan annuel suivant les modèles précis donnés par le FNBE. Ces municipalités devaient envoyer leur curriculum et le plan annuel de leurs écoles au FNBE, pour examen et validation. Pour mettre en œuvre le curriculum national, les élus, les chefs d'établissements et les enseignants furent formés lors de stages approfondis, effectués pendant leurs heures de service. Ces programmes étaient obligatoires pour tous les enseignants de l'école de base, ce qui permettait d'éviter que les enseignants ne se sentent fragilisés par la mise en œuvre de la réforme, même s'ils ne l'appréciaient pas forcément.

7 Le curriculum national pour l'enseignement fondamental a été réformé trois fois depuis 1970 et a été transformé en un core curriculum ${ }^{4}$ national qui définit des directives communes afin que toutes les municipalités et tous les établissements puissent faire ce qu'elles ont à faire. Objectifs et principes généraux aussi bien que syllabi plus détaillés ont désormais été inclus dans le même document. En 1985, le premier core curriculum national, publié par le FNBE, renforça la responsabilité des municipalités, en tant que fournisseurs d'offre d'éducation, ainsi que le rôle des curricula municipaux. On était fermement convaincu de la nécessité de mieux prendre en compte les spécificités des différentes municipalités dans l'organisation de l'enseignement. En 1994, on a délégué aux municipalités et aux écoles encore plus de pouvoir de décision, alors que, dans le même temps, seules des lignes très générales étaient définies dans le core curriculum national. Les curricula définis par les établissements ont gagné en importance et les enseignants ont participé de manière active à la mise au point de ces curricula, qu'ils soient d'établissement ou municipaux. Les élus, les chefs d'établissement et les enseignants ont également été utilisés comme experts par la DNEF dans le processus de définition du core curriculum national, participation qui exprimait ainsi toute la confiance accordée aux municipalités et aux établissements. Cette approche 
participative a eu un fort impact positif sur les connaissances des enseignants en matière de curriculum, de même que cela a accentué leur professionnalisme. Cela a également renforcé l'implication des élus locaux, des chefs d'établissement et des enseignants dans les objectifs définis par le core curriculum national (Halinen et Järvinen 2008).

En 2004, le statut du core curriculum national a de nouveau été renforcé après l'apparition, vers la fin de la décennie quatre-vingt-dix, de certaines inquiétudes en matière d'équité et d'égalité. La société finlandaise devenait en effet plus multiculturelle et plus hétérogène, et une récession économique avait aggravé la situation économique des municipalités, élargissant du même coup le fossé entre elles en matière d'éducation. Les municipalités et les établissements exprimèrent alors leur besoin de pouvoir disposer de directives nationales plus fortes. La réforme de 2004 a été évaluée en détail par le FNBE et par le Conseil finlandais de l'évaluation de l'éducation. Selon ces évaluations, la réforme a été un succès, et l'importance du core curriculum national ainsi que son influence sur le travail des enseignants semblent s'être accrues de manière significative (Kartovaara 2007).

\section{Caractéristiques principales du système actuel du curriculum}

9 Aujourd'hui, l'administration de l'éducation en Finlande est légère, flexible et vise à « encourager ». Son pilotage est fondé sur des buts et des objectifs nationaux communs clairement définis. Ceux-ci sont établis par la législation en matière d'éducation et par les core curricula nationaux.

10 Le Parlement finlandais prend des décisions en matière de législation sur l'éducation et de principes généraux de politique éducative. Le gouvernement, le ministère de l'Éducation et le FNBE sont chargés de la mise en œuvre de cette politique à l'échelle de la nation. Comme fournisseurs de l'offre d'éducation, les municipalités se voient déléguer un pouvoir considérable. Municipalités et écoles disposent d'une grande autonomie en matière d'organisation de l'enseignement et de mise en œuvre du core curriculum. Cette délégation de décision se fonde sur la confiance en la capacité des autorités municipales, des chefs d'établissement et des enseignants à faire face à leurs responsabilités. Quand le gouvernement participe aux frais scolaires, il le fait en déduction du transfert légal vers les municipalités.

11 Le système éducatif est régi par des lois et des décrets ainsi que par les décrets gouvernementaux en matière d'objectifs nationaux généraux et de répartition des heures de cours. Ces lois définissent les objectifs éducatifs communs, les principes selon lesquels l'enseignement doit être dispensé, ainsi que d'autres points, comme les matières principales enseignées à tous les élèves et la ventilation des heures d'enseignement suivant ces matières.

12 Il existe plusieurs core curricula: un pour l'enseignement pré-élémentaire, un pour l'enseignement de base, un pour l'enseignement secondaire supérieur général, un pour l'enseignement de base et secondaire supérieur pour adultes et enfin un pour les activités pré- et postscolaires destinées aux élèves de l'enseignement de base. Ces core curricula nationaux constituent la base administrative, juridique, intellectuelle et pédagogique à partir de laquelle se structure le travail des différents fournisseurs 
d'offre d'éducation. Ces derniers sont responsables de la conception des curricula locaux, qui peuvent être taillés sur mesure, soit au niveau de l'ensemble de la municipalité, soit pour chaque établissement, soit encore par une combinaison des deux (Kartovaara 2007, FNBE 2004).

Les core curricula et les curricula locaux concrétisent ensemble les lois et décrets en matière d'éducation, au sens administratif et juridique, et constituent des cadres administratifs plus précis permettant d'organiser l'enseignement dans les municipalités et les écoles. La mission intellectuelle des curricula est de choisir et de définir les connaissances et les compétences considérées comme suffisamment importantes pour devoir être maîtrisées par la génération suivante, et donc être enseignées. Pédagogiquement parlant, les curricula encouragent les enseignants et les autres personnels éducatifs à planifier leurs tâches et à choisir des méthodes de travail, tout en leur octroyant une autonomie professionnelle étendue. Ces encouragements sont le reflet de l'éthique finlandaise de valorisation de la profession d'enseignant ainsi que de la considération dont ils sont l'objet. Le système finlandais de gestion de l'éducation peut être illustré par un exemple tiré de l'enseignement fondamental (figure 1).

Fig.1 Gestion du curriculum en Finlande
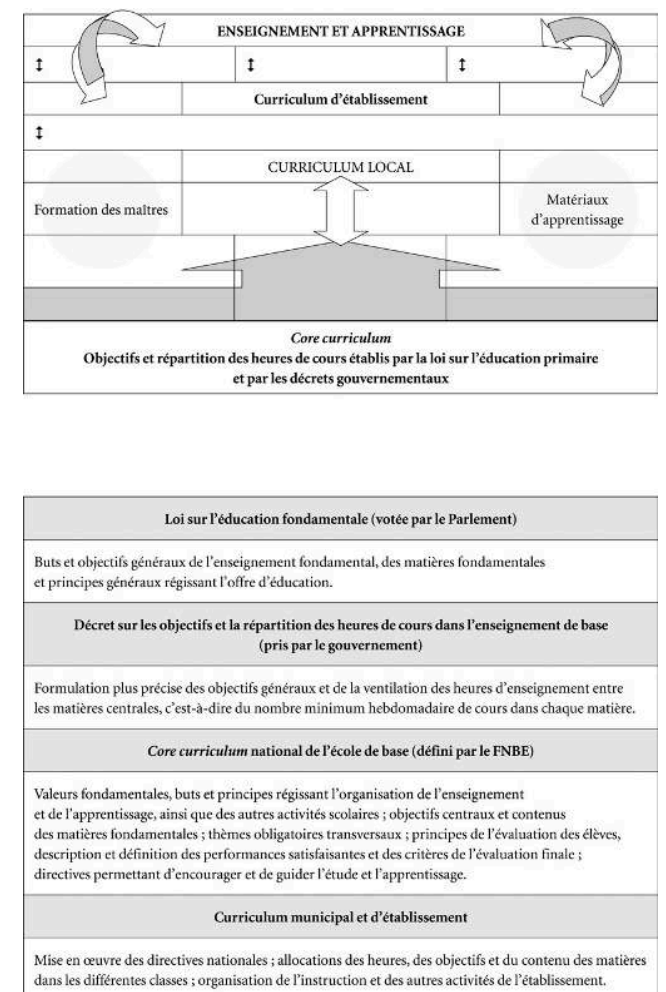

14 Les normes nationales, qu'il s'agisse des décrets-lois en matière d'éducation, de décrets gouvernementaux et de core curriculum, servent de directives communes pour l'ensemble des municipalités et les établissements et constituent les bases solides sur lesquelles toutes les parties peuvent s'appuyer pour planifier leur travail. L'élément le plus important du système est le processus d'enseignement et d'apprentissage au jour le jour, c'est-à-dire l'interaction entre les élèves et les enseignants. La qualité de ce processus est absolument essentielle pour atteindre les objectifs assignés à 
l'enseignement et pour satisfaire les différents besoins des élèves. Le système tout entier vise à favoriser ce processus, ainsi qu'à promouvoir le succès de l'apprentissage, une croissance saine et harmonieuse ainsi que le développement personnel de tous les élèves (Halinen 2006a).

La mise en œuvre du core curriculum est prise en charge dans la formation initiale et continue des enseignants, formation qui est dispensée principalement par les universités, ainsi que par les manuels et d'autres supports d'études produits par des éditeurs privés et par le FNBE. Ensemble, ces trois éléments - le curriculum, la formation des enseignants et les matériaux d'études - forment une base solide et cohérente pour le travail des enseignants.

$16 \mathrm{Au}$ lieu de se concentrer sur le contrôle, la finalité de l'ensemble du système est bien plutôt d'encourager et de développer le travail des municipalités et des établissements, c'est-à-dire de les aider à accomplir leur mission. Durant le cycle d'enseignement fondamental, il n'existe ni inspection d'établissement ni examens nationaux sanctionnant des résultats d'apprentissage, sur la base desquels les établissements pourraient être classés par ordre de mérite. Il n'existe aucun classement entre les écoles. L'accent est mis sur l'autoévaluation, la coopération et la transparence, et non sur la comparaison et la compétition entre établissements. L'autoévaluation des municipalités et des établissements est encouragée par l'évaluation des résultats de l'apprentissage à l'échelle nationale sur la base d'un échantillon représentatif, sous la supervision du FNBE. Elle est également encouragée par l'évaluation nationale de la santé et du bien-être des élèves, comme elle l'est par des évaluations thématiques externes, organisées par le Conseil finlandais de l'évaluation de l'éducation. Les informations collectées à partir de ces évaluations, tant au niveau national qu'au niveau municipal, sont utilisées à des fins d'amélioration de l'ensemble ainsi que pour la formation des enseignants. L'autoévaluation a également contribué à ce que les activités soient transparentes pour les parents et les autres parties prenantes et, ainsi, à faciliter une compréhension commune et en cohérence des finalités, des procédures et des résultats du système. La confiance mutuelle, la coopération et l'interaction sont les prérequis fondamentaux autour desquels se construit et se développe l'éducation finlandaise (Halinen 2006b).

\section{Le curriculum comme processus coopératif}

17 Dans le système éducatif finlandais, le curriculum est davantage un processus qu'un produit achevé. Avant tout, cela signifie que, sur l'ensemble des trois niveaux national, municipal et d'établissement -, les curricula sont créés au sein de processus extensifs et coopératifs. En second lieu, cela signifie que les core curricula (et curricula locaux) sont considérés comme des documents vivants, en constante évolution. Ils sont utilisés comme bases pour la préparation du travail quotidien et comme point de départ des améliorations en cours du système éducatif. Leur mise en œuvre est encouragée par différents programmes de développement et d'évaluation, tant au niveau national que municipal, programmes qui évoluent chaque fois que le demandant les différents contextes locaux.

Le FNBE définit les grandes lignes des core curricula en coopération avec de larges réseaux, composés de représentants des universités, et tout spécialement des départements de formation des maîtres, des associations d'enseignants et de 
municipalités, de représentants des éditeurs, des autorités municipales d'éducation, des chefs d'établissement et des enseignants, des parents et des élèves, ainsi que, comme le stipule la loi, des représentants de l'Agence nationale des affaires sociales et de la santé. Ces représentants sont invités à participer à des groupes de travail chargés de la conception des ébauches des core curricula. De nombreux séminaires et discussions sont également organisés. Lorsque le core curriculum de l'enseignement fondamental a été défini en 2004 sous la direction du FNBE, plus de deux cents municipalités et cinq cents établissements ont participé au processus. Ils ont joué le rôle d'alliés critiques, apportant de précieux retours sur les différentes ébauches du core curriculum. Au cours de ce travail préparatoire, les municipalités ont été soutenues et encouragées par l'intermédiaire d'un programme de formation intensive destiné aux coordinateurs des curricula locaux, et organisé par le FNBE. Ils purent ainsi se préparer au processus de création des curricula locaux, immédiatement après la finalisation du core curriculum national.

Dans le processus de définition du curriculum à l'échelle municipale et scolaire, le rôle des parents et des élèves est crucial. Leur opinion doit être prise en compte, tout spécialement lorsque sont débattus les valeurs, les objectifs, les règles communes et les modalités de fonctionnement. Les autorités municipales encouragent les écoles dans le processus de définition de leur curriculum et favorisent la coopération entre les différents groupes d'établissements et d'enseignants. Par l'intermédiaire d'autres acteurs locaux, tels que les travailleurs sociaux ou les intervenants auprès de la jeunesse, les centres de santé, les bibliothèques et musées, les galeries d'art, les clubs de sport, l'Église et les entreprises, le curriculum est enrichi et sa mise en œuvre renforcée, comme le sont les liens entre les établissements et la communauté environnante. Cette coopération extensive permet de s'assurer que toutes les parties ont une connaissance suffisamment approfondie des questions concernant le curriculum, et que différents points de vue y ont été intégrés. Par-dessus tout, enfin, cette coopération permet de s'assurer que les enseignants sont soutenus par les autres acteurs de la société (Halinen 2007, Halinen et Järvinen 2008).

Le curriculum d'établissement, lui, a plusieurs finalités. Il constitue la base du plan annuel de l'établissement, du projet pédagogique de chaque enseignant et de chaque membre du personnel scolaire, et enfin du projet d'études individuel des élèves. C'est une importante source d'informations pour les parents, pour les autres écoles et pour les autres secteurs de la communauté et, bien sûr, pour les autorités municipales. Les autorités nationales, elles, ne contrôlent plus les curricula municipaux ou d'établissement comme ils avaient l'habitude de le faire dans les décennies soixante-dix et quatre-vingt. Toutes sortes de procédures d'inspection et de ratification ont été abolies au début des années quatre-vingt-dix. À la place, les autorités nationales et les chercheurs utilisent les curricula locaux comme source d'information afin d'en tirer des conclusions relatives à la qualité et aux besoins d'amélioration du système.

\section{Une conception holistique du curriculum}

21 La conception du curriculum est holistique. Les core curricula nationaux, comme les curricula locaux, couvrent le champ entier de ce qui se passe à l'école, et non pas seulement les objectifs et les contenus des différentes matières. Sa conception est également inclusive, au sens où le même curriculum concerne l'éducation de tous les 
élèves, même ceux qui souffrent des handicaps les plus sévères (Halinen et Järvinen 2008). Ces deux caractéristiques sont importantes parce qu'elles définissent le rôle du curriculum, comme elles influencent la manière dont celui-ci sera mis en œuvre concrètement. L'approche holistique et la nature inclusive des core curricula comme des curricula locaux facilitent leur utilisation quotidienne dans les établissements. Peu à peu, les enseignants se sont habitués à prendre connaissance des finalités du curriculum, à planifier leur travail pédagogique et leurs matériaux d'apprentissage en fonction de celui-ci, sans se limiter à utiliser des manuels scolaires prêts à l'emploi. Les enseignants se sentent également responsables de la mise en œuvre des objectifs fixés par le curriculum, de manière à encourager et à promouvoir l'apprentissage et le bienêtre de chaque élève (Atjonen et al. 2008). Les core curricula de l'enseignement préscolaire, fondamental ou secondaire supérieur et adulte comprennent des directives pédagogiques communes. Ensemble, ils constituent le socle qui permet ensuite l'apprentissage tout au long de la vie.

22 Prenons à nouveau un exemple tiré de l'enseignement fondamental. Le core curriculum couvre tous les aspects importants du travail scolaire; il définit leurs grands objectifs opérationnels et indique les procédures par lesquelles ces objectifs et ces principes devraient se concrétiser dans les curricula locaux. Cela oblige les établissements à coopérer avec les parents et avec les autorités municipales sociales et sanitaires, tout spécialement en ce qui concerne les questions de développement et de bien-être des élèves. Cela oblige également les municipalités et les établissements à évaluer et à développer de manière permanente leur propre travail. Le core curriculum définit les valeurs fondamentales de l'enseignement, la conception de l'apprentissage et les critères qui président au choix des méthodes pédagogiques et de travail, ainsi que des directives permettant de développer un environnement favorisant l'apprentissage et la culture de travail de l'établissement. Il définit également les principaux contenus de l'enseignement et les modalités d'organisation de cet enseignement, du conseil en direction des élèves, des conditions de leur bien-être ainsi que des autres formes de soutien. Il définit, enfin, les grands principes de l'évaluation des élèves ainsi que le niveau caractérisant une bonne performance.

Les curricula locaux doivent se fonder sur le core curriculum national et doivent, concomitamment, prendre en compte les différences, les exigences et les opportunités locales, et tout spécialement les besoins des enfants et des familles de la zone considérée. Dans la plupart des cas, les autorités municipales délèguent les questions de curriculum aux établissements, et chaque établissement dispose d'un curriculum qui lui est propre. Les enseignants et autres personnels éducatifs sont fortement incités à participer au processus de planification du curriculum d'établissement. Lorsqu'ils débattent de ces questions, ils doivent intégrer tous les aspects fondamentaux pouvant influencer leur enseignement ou l'apprentissage des élèves. Ensemble, ils décident de la meilleure façon d'organiser le soutien face aux difficultés d'apprentissage, l'éducation multiculturelle et l'éducation adaptée aux besoins spécifiques; ils décident également de la manière dont le suivi individualisé des élèves est mis en place et dont ils s'assurent effectivement du bien-être des élèves qui leur sont confiés. Ils planifient la coopération entre les familles et l'école, et conçoivent la stratégie d'acquisition de connaissances qui définit la façon dont les TIC $^{5}$ et l'enseignement virtuel sont utilisés en cours, quel type de matériel sera nécessaire et comment le savoir-faire des enseignants et des autres personnels éducatifs en matière de TIC est développé. Ils 
conçoivent également un plan relatif à la -sécurité de l'environnement pédagogique, au suivi des absences des élèves et la façon dont on les protège des brimades, de la violence et du harcèlement (Halinen 2007).

Lors du processus de planification du curriculum, les enseignants et les autres personnels d'éducation apprennent à considérer le fonctionnement de leur établissement comme une entité, tout en s'engageant eux-mêmes à être responsables de l'ensemble, et non pas seulement de leur classe ou de leur matière. Ce faisant, leur expertise et leur professionnalisme se voient fortement développés.

\section{Défis à venir}

Tout autour de l'école, le monde change rapidement. De nouveaux besoins d'apprentissage apparaissent, et même les meilleurs systèmes éducatifs doivent évaluer leur situation actuelle, considérer de manière ouverte les nouveaux défis qui se présentent et être prêts à procéder aux ajustements indispensables.

Le débat actuel en Finlande touche des questions telles que: "Comment le monde change-t-il? Quels sont les changements les plus importants en ce qui concerne l'éducation? En conséquence, de quels types de connaissances et de compétences avons-nous besoin ? Lorsque les connaissances elles-mêmes changent si rapidement, comment définir le contenu pertinent d'un cycle d'études? Comment devrions-nous développer les processus pédagogiques et prendre en compte l'apprentissage qui se produit en dehors de l'école? Comment renforcer le rôle actif des apprenants? Comment utiliser le temps scolaire de la meilleure manière possible, et comment mettre en relation les apprentissages formel et informel ? (MOE 2010).

Ces questions, et d'autres, devraient trouver une solution très rapidement. Les réponses que nous apporterons influenceront l'ensemble du système, les questions de curriculum, ainsi que l'organisation concrète du travail dans les écoles. Nous avons besoin d'une réflexion encore plus affûtée et plus holistique sur les curricula, là où les dimensions principales de ce curriculum - les objectifs, les contenus et la mise en œuvre - forment une entité systémique, équilibrée et soigneusement planifiée. L'accent devrait porter tout particulièrement sur les objectifs et les processus pédagogiques, et non pas tant sur les contenus, puisque ceux-ci peuvent très bien rapidement perdre de leur pertinence. Au lieu de cela, les objectifs, définis en termes de compétences - telles que la réflexion et la résolution de problèmes, le travail et l'interaction, l'expression au moyen de différents outils, la participation et la prise d'initiative, etc. -, ainsi que les processus qui président à la formation de ces compétences, sont de la plus extrême importance pour l'avenir.

En 2009, prenant en compte des questions telles que le changement climatique, la mondialisation et ses effets sur la société finlandaise, les défis économiques, le développement technologique et tout spécialement le rôle des TIC, les changements dans la création et la médiation des connaissances, ainsi que ceux apportés à la structure familiale et au processus de développement des enfants, le ministère de l'Éducation a initié un travail préparatoire à une nouvelle réforme de l'enseignement fondamental (MOE 2010). Ce travail affectera également le core curriculum. Cette réforme devrait être mise en place entre 2012 et 2014. Le débat politique très fourni et les très nombreuses discussions publiques auxquelles cette réforme a donné lieu sont le 
signe de l'importance croissante des questions d'éducation et de curriculum dans la société finlandaise.

\section{BIBLIOGRAPHIE}

ATJONEN P., et al. (2008) : Tavoitteista vuorovaikutukseen; Perusopetuksen pedagogiikan arviointi. (From Goals to Interaction; Evaluation of the Pedagogy in Basic Education). Koulutuksen arviointineuvoston julkaisuja 30. Report of the Finnish Education Evaluation Council, $\mathrm{n}^{\circ} 30$. Committee on the Comprehensive School Curriculum (1970): Committee Deliberations I. Opetussuunnitelman perusteet. Komiteanmietintö 1970:A5, Helsinki: Kouluhallitus.

FNBE (Finnish National Board of Education) (2004a): National Core Curriculum for Basic Education. Helsinki: Finnish National Board of Education.

HALINEN I. (2006a): The Finnish Curriculum Development Process. In A. Crisan (Ed.), Current and future challenges in curriculum development; Policies, practices and networking for change (p. 62-78). Center Education 2000+ and UNESCO/IBE. Bucharest : Humanitas Educational.

HALINEN I. (2006b) : L'école de l'équité et de l'intégration : le cas de la Finlande. Revue internationale d'éducation Sèvres, $\mathrm{n}^{\circ} 41$.

HALINEN I. (2007): Der Lehrplan der Gemeinschaftsschule und die Weiterentwicklung der Schulausbildung in Finnland (Comprehensive school curriculum and educational development in Finland). In J. Sarjala \& E. Häkli (Eds.), Jenseits von Pisa: Finnlands Schulsystem und seine neuesten Entwicklungen (p. 99-122) Berlin: Berliner Wissenschafts-Verlag.

HALINEN I. \& JÄRVINEN R. (2008): Towards inclusive education: the case of Finland. Prospects 145, Vol $38, \mathrm{n}^{\circ} 1$, mars (p. 77-97). UNESCO.

HAUTAMÄKI J. et al. (2008). PISA 06 Finland ; Analyses, reflections and explanations. Ministry of Education, Publications 2008:44. Helsinki: Helsinki University Print.

KARTOVAARA E. (Ed.) (2007): Perusopetuksen vuoden 2004 opetussuunnitelmauudistus (Reform of the 2004 core curriculum for basic education). Helsinki: Opetushallitus.

MOE. Ministry of Education and Culture (2010): Perusopetus 2020 - yleiset valtakunnalliset tavoitteet ja tuntijako. (Basic Education 2020 - the national general objectives and distribution of lesson hours; a committee proposal). Opetus- ja kulttuuriministeriön työryhmämuistioita ja selvityksiä 2010 :1. Helsinki : Yliopistopaino.

\section{NOTES}

1. «Comprehensive schools » dans le texte : l'école de base finlandaise s'adresse à tous les élèves de 7 à 16 ans. (NdlR).

2. école de base, mais aussi « comprehensive schools ", c'est-à-dire unifiées et ouvertes à tous.

3. Liste des sujets enseignés dans chaque discipline (NdlR). 
4. Le terme désigne un tronc commun obligatoire autour duquel peuvent être proposés des enseignements optionnels ( $\mathrm{NdlR}$ ).

5. Technologies de l'information et des communications (NdT).

\section{RÉSUMÉS}

Cet article examine le rôle central du curriculum dans la gestion et le développement de l'enseignement en Finlande. Ce rôle est rattaché à la conception holistique du curriculum, à la façon dont ce curriculum est créé grâce à la coopération intense de différents acteurs, et comment il est activement utilisé à différents niveaux du système éducatif. L'article se concentre sur l'enseignement général et, à l'intérieur de celui-ci, sur l'enseignement dit fondamental. Il examine le développement historique du curriculum depuis les années 1970, et la façon dont ce dernier s'articule aux changements sociétaux. Enfin, l'auteur se penche sur les défis auxquels doit faire face le double développement du curriculum et du système éducatif.

The article examines the central role of curriculum in the management and development of education in Finland. This role is connected to the holistic conception of curriculum, how curriculum is created by intensive cooperation of different stakeholders, and how it is actively used at different levels of the education system. The article focuses especially on general education, and within that on basic education. It looks also shortly at the historical development of the curriculum since the 1970's, and how this development is connected to the changes in society. Finally, the writer reflects the actual challenges in the development of both the curriculum and the education system.

Este artículo examina el papel primordial del currículum en la gestión y el desarrollo de la enseñanza en Finlandia. Dicho papel está vinculado con la concepción holística del currículum, con la forma en que éste ha sido creado gracias a la intensa cooperación de diferentes actores, y cómo es activamente utilizado en los diferentes niveles del sistema educativo. El artículo se centra en la enseñanza general y más particularmente, la enseñanza denominada fundamental. Examina el desarrollo histórico del currículum desde los años 1970 y su vínculo con los cambios societales. Por último, el autor analiza los desafíos que deberán enfrentar tanto el desarrollo del currículum como el del sistema educativo.

\section{INDEX}

Palabras claves : conocimiento, contenido de la educación, curriculum, historia de la educación, programa de enseñanza, calidad de la educación, calidad de la enseñanza

Index géographique : Finlande

Keywords : learning, education content, curriculum, educational history, instructional programmes, educational quality, teaching quality

Mots-clés : connaissance, contenu de l'éducation, curriculum, histoire de l'éducation, programme d'études, qualité de l'éducation, qualité de l'enseignement 


\section{AUTEUR}

\section{IRMELI HALINEN}

Irmeli Halinen dirige l'Unité de développement du curriculum, au Conseil national de l'Éducation en Finlande. Elle travaille depuis près de quarante ans sur les questions d'évaluation et de développement. Elle est membre du Conseil national de l'évaluation de l'éducation en Finlande et de plusieurs organismes privés et publics, finlandais et internationaux, auxquelles elle apporte son expertise en matière de développement curriculaire notamment. Ancienne enseignante et chef d'établissement, elle a également été responsable de l'amélioration de la qualité de l'école pour la Ville de Helsinki. Elle a publié de nombreux articles, tant en Finlande qu'à l'étranger. 